\title{
Quadratic Jordan Triple Systems
}

\author{
Amir Baklouti \\ Correspondence: Amir Baklouti, Umm Al-Qura University, College of preliminary year, Department of mathematics, \\ P.O. Box 14035, Makkah Al-Mukarramah 21955, Saudi Arabia \& Univeristé de Sfax, IPEIS-Route Menzel Chaker Km \\ 0, 5-3018 Sfax Tunisie. BP 1172-3018 Sfax
}

\author{
Received: January 8, 2019 Accepted: February 11, 2019 Online Published: March 4, 2019 \\ doi:10.5539/jmr.v11n2p68 URL: https://doi.org/10.5539/jmr.v11n2p68
}

\begin{abstract}
In this work, We show that every Jordan triple system can be viewed as a $T^{*}$ extension of another one or an ideal of co-dimension one of a Jordan triple system whose represent the $T^{*}$ extension of another Jordan triple system. Moreover, several result involving the structure of quadratic Jordan triple systems are given.
\end{abstract}

Keywords: Jordan triple systems, Jordan algebras, Lie algebras

\section{Introduction}

The triple system structures play an important role in various domains of theoretical and mathematical physics as for example Nambu mechanics (Nambu, 1973; Takhtajan, 1994). Furthermore, other motivation to study triple systems comes from string theory and M-branes involving naturally an algebra with a ternary operation (Bagger \& Lambert, 2007). This give another motivation for development of mathematical concepts such as Jordan triple systems.

It is well known that using the T-K-K construction, we can construct a Lie triple system starting a Jordan one Moreover, from a Lie triple system, we can obtain a graded Lie algebra.

In this paper, we start by giving some preliminary results. After that, we extend the notion of $T^{*}-$ extention, introduced by M. Bordemann in case of nonassociative algebras, to contruct a non semisimple Jordan triple system. Recall that M. Bordemann shows in his work that if the Jordan algebra have an even dimension which have an isotropic ideal of dimension equal to half of the dimension of the algebra, then the original algebra is a $T^{*}$-extension of another Jordan algebra. In the case where the dimension of the Jordan algebra is odd, this Jordan algebra comes an ideal of codimension one of a $T^{*}$-extension. An extension of this result to the case of Lie triple system can be found. One of the aims of this paper is to extend these results to the case of Jordan triple system. We shall proceed differently from the Jordan algebra case. We shall use the relashinship between Jordan triple systems and Lie triple systems.

Definition 1. We define Jordan triple system as a linear space A endowed with a trilinear map $\{u, v, w\}$ which satisfy

$$
\begin{aligned}
& \{u, v, w\}=\{w, v, u\} \\
& \{x, y,\{u, v, w\}\}-\{u, v,\{x, y, w\}\}=\{\{x, y, u\}, v, w\}-\{u,\{y, x, v\}, w\} .
\end{aligned}
$$

Definition 2. A Linear map D of A is called a derivation if it satisfy $D$ of $A$ into $A$ such that

$$
D(\{u, v, w\})=\{D(u), v, w\}+\{u, D(v), w\}+\{u, v, D(w)\}, \forall u, v, w \in A .
$$

The set containing the derivation of A will be denoted by Der $(A)$. This set is a Lie algebra and called the derivation algebra of $A$. An example of derivation of Jordan triple systems $A$ can be defined for $u, v \in A$, by the map $L(u, v)(r e s p . R(u, v))$ which are defined by $L(u, v) w=(u, v, w)$ ( resp. $R(u, v) w=(w, u, v))$, for all $w$ in $A$, is a derivation of $A$ These maps are called respectively the left and the right multiplication. The sub-algebra $L(T, T)=\left\{\sum_{a_{i}, b_{i}} L\left(a_{i}, b_{i}\right) ; a_{i}, b_{i} \in A\right\}$ of Der $(A)$, is called The algebra of inner derivation .

Definition 3. (1) An invariant bilinear form $B$ on $A$ is a bilinear form who

$$
B(R(x, y) z, u)=B(z, R(y, x) u) \text { and } B(L(x, y) z, u)=B(z, L(y, x) u) \text { for all } x, y, z \in A .
$$

If a symmetric bilinear form $B$ is right invariant or left invariant, then B is left invariant also. That is, B the invariance of $B$ is equivalent to the fact that $B$ is right invariant.

(2) A quadratic Jordan triple system is a Jordan triple system with a symmetric nondegenerate and invariant bilinear form on B. Such bilinear form is said a quadratic structure on A. If $(A, B)$ is a quadratic Jordan triple system which 
contain an ideal where the restriction of $B$ onto this ideal is nondegenerate, we say that $(A, B)$ is reducible. Elsewhere, $(A, B)$ is said irreducible. (3)A symplectic form is a skewsymmetric bilinear form $\omega$ satisfiying

$$
\omega(u,\{t, w, v\})-\omega(v,\{t, w, u\})+\omega(w,\{v, u, t\})+\omega(t,\{u, v, w\})=0,
$$

$\forall u, v, w, t$ in A. A Jordan triple system endowed with a symplectic form is called symplectic Jordan triple system.

Definition 4. A symmetric, (resp. antisymmetric) homomorphism of $A$ with respect to $B$ is a homomorphism $g$ whose satisfies if $B(g(u), v)=B(u, g(v)),($ resp. $B(g(u), v)=-B(u, g(v)))$, for all $u, v$ belonging to $A$.

The following theorem give a necessary and sufficient condition for making a symplectic Jordan triple system quadratic.

Theorem 0.1. A symplectic Jordan triple system $(A, \omega)$ have a quadratic structure $B$ if and only if there is a derivation $D$ of $A$ which is invertible and antisymmetric with respect to $B$ satisfying $\omega(u, v)=B(D(u), v)$ for all $u, v$ belonging to $A$.

Proof. Let $D$ be an invertible and antisymmetric Derivation with respect to $B$. It is is easy to check that the bilinear form $\omega$ of $A$ defined by: $\omega(u, v)=B(D(u), v), \forall u, v \in A$, is symplectic.

Conversly,It is well known that if $\omega$ and $B$ are two nondegenerate bilinear forms, then there is a homomorphism $D$ of $A$ which satisfies $\omega(u, v)=B(D(u), v), \forall u, v \in A$. Furthermore, the fact that the bilinear form $\omega$ is a symplectic form implies that

$$
B(D(\{u, v, w\}), t)=B(\{D(u), v, w)\}, t)+B(\{u, \delta(v), w)\}, t)+B(\{u, v, \delta(w))\}, t) A .
$$

Since the bilinear form $B$ is non-degenerate, then $D$ becomes a derivation.

now, we are ready to announce the theorem of construction of quadratic Jordan triple system from a non necessary quadratic one.

Theorem 0.2. Onsider a Jordan triple system $(A,\{,\}$,$) and a trilinear map \Omega$ from $A \times A \times A$ onto $A^{*}$. On the linear space $T_{\Omega}^{*}(A)=A \bigoplus A^{*}$ we define the following multiplication

$$
\{u+f, v+g, w+h\}=\{u, v, w\}_{A}+\Omega(u, v, w)+\{f, v, w\}+\{u, g, w\}+\{u, v, h\},
$$

for all $u, v, w \in A$ and $f, g, h \in A^{*}$. Where,

$$
\{f, v, w\}(t)=f(\{t, w, v\}) ; \quad\{u, g, w\}(t)=g(\{u, t, w\}) \quad \text { and }\{u, v, h\}(t)=h(\{v, u, t\}), .
$$

The linear space $T_{\Omega}^{*}(A)$ endowed with the multiplication above is an Jordan triple system if and only if, the map $\Omega$ verify the following identity:

$$
\Omega(u, v, w)=\Omega(w, v, u)
$$

and

$$
\Omega(a, b,\{u, v, w\})-\Omega(u, v,\{a, b, w\})=\Omega(\{a, b, u\}, v, w)-\Omega(u,\{b, a, v\}, w) .
$$

$\forall a, b, u, v, w \in A$. Moreover, if we define on $T_{\Omega}^{*}(A)$ the bilinear form $B$ by:

$$
B(u+f, v+g)=g(u)+f(v)
$$

then $B$ is a symmetric nondegenerate and invariant bilinear form on $T_{\Omega}^{*}(A)$.

$\left(T_{\Omega}^{*}(A), B\right)$ constructed above is said the $T_{\Omega}^{*}$-extension of $A$ by $\Omega$.

Proof. Calculation.

Theorem 0.3. Every $T^{*}$-extension of a Jordan triple system admitting an invertible derivation $D$ admits a symplectic structure.

Proof. The result follows from Theorem 0.1. 
Example 0.1. Let $A$ be a Jordan triple system and $G_{p}=x \mathbb{K}[x] / t^{p} \mathbb{K}[x]$, where $p$ is a not nul positive integer. On the linear space $A_{p}=A \otimes G_{n}$ we define the following product:

$$
\left\{x \otimes u^{q}, v \otimes x^{s}, w \otimes x^{t}\right\}=\{u, v, w\} \otimes t^{q+s+t},
$$

with $u, v, w$ belong to $A$ and $q, s, t$ are not null posive integers.

We get that $A_{n}$ is a Jordan triple system which is nilpotent. The homomorphism $D$ of $A_{p}$ :

$$
D\left(x \otimes x^{q}\right)=q\left(x \otimes x^{q}\right)
$$

for any $x \in A$ and $q$ is an integer smallest than $p$ is a derivation of $A_{p}$ which is invertible. Now, on the linear space $T^{*}$-extension of $T_{p}$ is a quadratic Jordan triple system. Moreover, this quadratic Jordan triple system endowed with the following derivation: $\tilde{D}(X+f)=D(X)-f \circ D$. Then $\tilde{D}$ is a symplectic Jordan triple system.

Proposition 0.4. Let $Z(A)=\{u \in A ;\{u, v, w\}=0, \forall v, w \in A\}$ be the center of $A$. Then, the orthogonal of $Z(A)$ with respect to $B$ is equal to the aasociator of the Jordan triple system $A$ which is the linear space spanned by $\{\{u, v, w\}, u, v, w \in A$.

Proof. Computation

Definition 5. For a Jordan triple system $A$ we denote by the ascending series $\left(A^{(p)}\right)_{p \in 0,1,2 \ldots}$ by $A^{(0)}=A$ and $A^{(p+1)}=$ $\left(A^{(p)}, A^{(p)}, A^{(p)}\right), \forall p \in \mathbb{N}$. By a same way, we define the descending serie $\left(A^{p}\right)_{p \in\{0,1,2 \ldots\}}$ by $A^{0}=A$ and $A^{p+1}=\left(A^{p}, A^{p}, A^{\prime}\right)$, $\forall p \in \mathbb{N}$ The Jordan triple system $A$ is said to be nilpotent (resp. solvable) If there is an integer $p$ satisfying $A^{(p)}=\{0\}$ ) $\left(\right.$ resp. $\left.A^{p}=\{0\}\right)$

Definition 6. Let $(A, B)$ be quadratic Jordan triple system

1. An ideal I of $A$ is a subspace of A satisfying $(I, A, A)+(A, I, A) \subseteq I$.

2. $\mathcal{U}$ of $A$ is called:

(a) Nilpotent (resp. solvablet) if it is nilpotent (resp. solvable) as a Jordan triple system.

(b) Degenerate (resp. non-degenerate) if $B_{\mid l \times l}$ is degenerate (resp. non-degenerate).

(c) Abelian if $\{I, I, I\}=\{0\}$.

3. The biggest solvable ideal of $\mathcal{V}$ is called the Jacobson radical of $\mathcal{V}$.

4. A Jordan triple system $(A, B)$ is semisimple if it contains no non trivial solvable ideal.

5. A B-irreducible Jordan triple system B-irreducible is a Jordan triple system whose contains no non trivial nondegenerate ideal

The proof of the following proposition is easy.

Proposition 0.5. The orthogonal with respect of $B$ of an ideal I of a quadratic Jordan triple system $(A, B)$ is an ideal of A. Moreover, if I is nondegenerate, then its orthogonal is also nondegenerate.

Lemma 0.6. Every quadratic Jordan triple system can be written as a direct summation of a nondegenerate, B-irreducible ideals $\left(A_{i}\right)_{i \in\{1, \ldots, r\}}$ which satisfies

$$
B\left(A_{i}, A_{j}\right)=\{0\}, \quad \forall i \neq j .
$$

Proof. To prove this lemma it is sufficient to precede by induction on $n=\operatorname{dim}(A)$. If the lemma is hold for any Jordan triple system of dimension $k$ less than $n$, then by the previous proposition, the lemma hold for $n$.

Lemma 0.7. In the case where A is semisimple, the previous decomposition coincide with the decomposition of A onto simple ideals.

Proof. Straitforward computation

Theorem 0.8. Every quadratic Jordan triple system of dimension $n$ where $n n$ is an even integer and containing an $\frac{n}{2}$ dimentional ideal which is isotropic is isomorphic to a $T^{*}$-extension of another Jordan triple system. 
Proof. The isotropic ideal $I$ of dimension $\frac{n}{2}$ is abelian because $B$ is nondegenerate. Its complementary isotropic ideal is denoted $V$

Let $u, v, w \in V$. We can write $\{a, b, c\}=\gamma(a, b, c)+\sigma(a, b, c)$, whith $\gamma(a, b, c) \in I$ and $\sigma(a, b, c) \in V$. We can easly verify that $(V, \sigma)$ is a Jordan triple system.

Consider the linear map $\mu: I \longrightarrow V^{*} ; i \longmapsto B(i,$.$) . This linear map is bijective. Moreover, \operatorname{dim}(I)=\frac{n}{2}=\operatorname{dim}\left(V^{*}\right)$. Thus, $\mu$ is an linear spaces isomorhism. Put the $T^{*}$-extension $T_{\Omega}^{*}(A)=V$ bigoplus $V^{*}$ of $V$ by $\Omega$ defined on $V$ by: $\Omega(u, v, w)=\mu\left(\gamma\left(u, v, w u, v, w \in V\right.\right.$. Moreover, let us consider $\Psi: A=I \bigoplus V$ longrightarrow $V \bigoplus V^{*} ; j+u \longmapsto u+\mu(j)$. The fact that $B$ is invariant implies that for all $u, v, w \in V, j \in I$,

$$
\mu(j)(\{u, v, w\})=B(j,\{u, v, w\})=B(\{j, w, v\}, u)=\mu(\{j, w, v\})(u) .
$$

Similarly,

$$
\mu(j)(\{u, v, w\})=\mu(\{w, j, u\})(v)=\mu(\{v, u, j\})(w) .
$$

Hence, if $\Psi: A=I \oplus V \longrightarrow V \bigoplus V^{*}$ defined by $j+u \longmapsto u+\mu(j), \forall j \in I, u \in V$ then, for $U=j+u, V=k+v, W=$ $p+w \in A=I \oplus V$, we obtain

$$
\begin{aligned}
\Psi(\{U, V, W\}) & =\sigma(u, v, w) \oplus \mu(\gamma(u, v, w))+\mu(\{u, v, p\})+\mu(\{j, v, w\})+\mu(\{u, k, w\}) \\
= & \sigma(u, v, w) \oplus \omega(u, v, w)+\mu(p)(\{v, u, .\})+\mu(j)(\{., w, v\})+\mu(k)(\{w, . ., u\}) \\
= & \{\Omega(U), \Omega(V), \Omega(W)\} .
\end{aligned}
$$

Which I;plies that $\Omega$ a Jordan triple systems isomorphism

Theorem 0.9. Every quadratic Jordan triple system of odd dimension $n$ containing an $\frac{n-1}{2}$ dimentional ideal which is isotropic is isometric to an ideal of dimension $n-1$ of a $T^{*}$-extension of another Jordan triple system.

Proof. Similar to the previous proof.

\section{Acknowledgements}

The authors would like to thank Deanship of Scientific Research at Umm Al-Qura University (Project ID 15-SCI-3-10025) for the financial support.

\section{References}

Bagger, J., \& Lambert, N. (2008). Gauge symmetry and supersymmetry of multiple M2-branes. Physical Review D, 77(6), 065008.

Baklouti, A. (2017). Quadratic Hom-Lie triple systems. Journal of Geometry and Physics, 121, 166-175. https://doi.org/10.1016/j.geomphys.2017.06.013

Baklouti, A., \& Benayadi, S. (2015). Pseudo-euclidean Jordan algebras. Communications in Algebra, 43(5), 2094-2123. https://doi.org/10.1080/00927872.2014.888562

Jacobson, N. (1949). Lie and Jordan triple systems. American Journal of Mathematics, 71(1), 149-170. https://doi.org/10.2307/2372102

Arnlind, J., Makhlouf, A., \& Silvestrov, S. (2010). Ternary Hom-Nambu-Lie algebras induced by Hom-Lie algebras. Journal of Mathematical Physics, 51(4), 043515.

Kantor, I. L. V. (1964). Classification of irreducible transitive differential groups. In Doklady Akademii Nauk, 158(6), 1271-1274. Russian Academy of Sciences.

Koecher, M. (1967). Imbedding of Jordan algebras into Lie algebras. I. American Journal of Mathematics, 89(3), 787-816. https://doi.org/10.2307/2373242

Nambu, Y. (1995). Generalized hamiltonian dynamics. In Broken Symmetry: Selected Papers of Y Nambu (pp. 302309). https://doi.org/10.1142/9789812795823_0027 Takhtajan, L. (1994). On foundation of the generalized Nambu mechanics. Communications in Mathematical Physics, 160(2), 295-315. https://doi.org/10.1007/BF02103278

Zhang, Z. X., Shi, Y. Q., \& Zhao, L. N. (2002). Invariant symmetric bilinear forms on Lie triple systems. Communications in Algebra, 30(11), 5563-5573. https://doi.org/10.1081/AGB-120015671 


\section{Copyrights}

Copyright for this article is retained by the author(s), with first publication rights granted to the journal.

This is an open-access article distributed under the terms and conditions of the Creative Commons Attribution license (http://creativecommons.org/licenses/by/4.0/). 